\title{
Gender differences in DNA damage/repair after laser-generated ultrafast electron beam irradiation
}

\begin{abstract}
The laser-driven particle acceleration technology has been developed over the last decade as a possible alternative to conventional particle accelerators. The significantly smaller size and cost, as well as the precise beam orientation compared with conventional cyclotrons ensure the potential application of laser-driven particle irradiation in imaging and treatment of cancerous cells. However, the radiobiological studies on laser-generated particle bunches are still required to promote its application in clinical practice. The aim of the work was to investigate the laser-generated ultrafast electron beam irradiation effect on DNA damage and repair in human peripheral blood mononuclear cells (PBMCs) with the respect to gender-specific radiosensitivity. The single cell gel-electrophoresis (comet) assay was used to analyze the level of DNA damage/repair. The level of DNA damages after irradiation was almost 3 times higher in female PBMCs, than that of in male PBMCs. The reparation capacity in female PBMCs was also lower, compared to male PBMCs. So, the difference between male and female DNA damage/repair response to laser-generated ultrafast electron beam irradiation was investigated. The gender bias in radiosensitivity was revealed, demonstrating higher female sensitivity to irradiation.
\end{abstract}

Keywords: laser-generated ultrafast electron beam, gender, DNA damage, repair, PBMCs
Volume 5 Issue 2 - 2018

\author{
Babayan N, ${ }^{1,2}$ Grigoryan B, ${ }^{3}$ Hovhannisyan \\ G, ${ }^{2}$ Tadevosyan G,' Khondkaryan L,' \\ Grigoryan R,' Sarkisyan N,' Aroutiounian R' \\ 'Group of Cell Technologies, Institute of Molecular Biology NAS \\ of RA, Armenia \\ ${ }^{2}$ Department of Genetics and Cytology, Yerevan State University, \\ Armenia \\ ${ }^{3}$ Advanced Research Electron Accelerator Laboratory, CANDLE \\ Synchrotron Research Institute, Armenia
}

\author{
Correspondence: Babayan Nelly, Institute of Molecular \\ Biology NAS of RA, Hasratyan 7, 0014 Yerevan, Armenia, Tel \\ +3749I-572573, Fax +37410-282061, \\ Email n babayan@mb.sci.am
}

Received: January 20, 2018 | Published: March 21, 2018
Abbreviations: AREAL, advanced research electron accelerator laboratory; CANDLE, center for the advancement of natural discoveries using light emission; PBMCs, peripheral blood mononuclear cells; OTM, olive tail moment; Gy, gray; StDev, standard deviation; h, hour

\section{Introduction}

The laser-generated electron acceleration is a developing technology, which can make a revolution in radiation therapy of tumors. The possibility of creating directed ultra-short pulses of enormous capacity (up to $\mathrm{kGy} / \mathrm{s}$ ) allows the precise dose-control induction of local effects on solid tumors with minimal exposure of normal tissues. The obvious advantage of laser-generated electron accelerators is also the significantly smaller size and cost than that of conventional radiotherapy machines. ${ }^{1}$ There are only a few publications on the biological effects of ultra-short pulsed electron beam radiation. ${ }^{2-5}$ However, before translating the usage of lasergenerated accelerators into clinical practice, the radiobiological effectiveness of such ultra-short electron bunches should be approved.

In clinical radiobiology gender-dependent radiosensitivity can have an impact on tumour response during radiation therapy, as well as on early and late side effects, including the induction of secondary malignancies. There are sporadic studies on the potential molecular and cellular bases for sex-specific radioreactions. Recently, the significant differences in radiosensitivity, associated with genetic polymorphic variations, was shown between females and males. ${ }^{6}$ The gender differences influence the response of platelets in mice following exposure to total body irradiation. ${ }^{7}$ The recent report on mortality among atomic bomb survivors shows that the excess relative risk of cancer is far higher for females than males. ${ }^{8}$ However, the biological and molecular bases of the sex-specific radioreactions are still need to be investigated.

The aim of the work was to investigate the effect of lasergenerated ultrafast electron beam irradiation on DNA damage and repair in human male and female peripheral blood mononuclear cells (PBMCs) using single cell gel-electrophoresis (comet) assay. Radiation treatment was carried out using $3.6 \mathrm{MeV}$ electron beam generated by a laser-driven radiofrequency gun-based linear AREAL accelerator at the CANDLE synchrotron research institute, Armenia. ${ }^{9}$

\section{Conclusion}

The effect of ultrafast electron beam irradiation on DNA damage and repair was studied using comet assay in human PBMCs (4 female and 4 male). The averaged data presented in the Table 1 demonstrated the dose-dependent increase of the primary DNA damage level started from $2 \mathrm{~Gy}$ up to the $8 \mathrm{~Gy}$ of irradiation ( $0 \mathrm{~h}$ time point). The subsequent DNA repair was evident 24hours after irradiation, since the level of DNA damages significantly decreased in comparison to corresponding data at $0 \mathrm{~h}$ time point.

Two-way ANOVA analysis was performed to compare radiosensitivity of male and female groups. As it is shown on the Figure 1, the gender bias in radiosensitivity was revealed. The gender effect on radiation-induced DNA damage/repair was considered extremely significant after adjustment with Bonferroni correction $(\mathrm{p}<0.0001)$. The female high radiosensitivity was demonstrated, since the DNA damage level in female PBMCs increased more than 4 times (8Gy) in comparison with control (Figure 1A). After 24h of incubation the reduction of DNA-damage was evident, but the overall level of DNA damage still was significantly higher than that of in control (Figure 1B). Male PBMCs are less radiosensitive, than female as even at the highest dose of irradiation (8Gy) the level of DNA damages increased less than 1,5 times in comparison with control (Figure 1A), and those damages were repairable (Figure 1B). So, the preliminary data on difference between male and female response to laser-generated ultrafast electron beam irradiation was investigated based on DNA damage/repair parameters in PBMCs. The gender bias in radiosensitivity was revealed, demonstrating higher female sensitivity to irradiation. 
Table I The level of DNA-damage in peripheral blood mononuclear cells after 0 and 24 hours of irradiation with ultrafast electron beam at the 3,6Gy/min dose-rate

\begin{tabular}{|c|c|c|c|c|c|c|}
\hline \multirow{3}{*}{ Dose, Gy } & \multicolumn{6}{|c|}{ OTM value (Mean \pm Stdev) } \\
\hline & \multicolumn{2}{|l|}{ Male } & \multicolumn{2}{|l|}{ Female } & \multicolumn{2}{|l|}{ Total } \\
\hline & $O h$ & $24 \mathrm{~h}$ & $\mathrm{Oh}$ & $24 \mathrm{~h}$ & $O \mathrm{~h}$ & $24 \mathrm{~h}$ \\
\hline 0 & $6,05 \pm 0,87$ & $3,83 \pm 1,02$ & $8,13 \pm 0,94$ & $5,09 \pm 1,52$ & $7,09 \pm 1,39$ & $4,46 \pm 1,37$ \\
\hline 2 & $6,39 \pm 0,92^{*}$ & $4,66 \pm 1,25$ & $|I, 8 \pm I, 7| *$ & $9,89 \pm 2,65^{*}$ & $9,10 \pm 3,16 *$ & $7,28 \pm 3,38^{\#}$ \\
\hline 4 & $5,84 \pm 0,84 *$ & $2,60 \pm 0,69$ & $19,09 \pm 2,77^{*}$ & $8,08 \pm 2,16 *$ & $12,47 \pm 7,33^{*}$ & $5,34 \pm 3,28^{\#}$ \\
\hline 8 & $9,80 \pm 1,42 *$ & $2,84 \pm 0,76$ & $32,18 \pm 4,66 *$ & $9,4 I \pm 2,52 *$ & $20,99 \pm 12,38^{*}$ & $6,12 \pm 3,\left.9\right|^{\#}$ \\
\hline
\end{tabular}

$*_{-p}<0.05$ in comparison to control; \# -p<0.05 in comparison to corresponding data at 0 h time point.

OTM, olive tail moment (the relative amount of DNA in the tail of the comet multiplied by the median migration distance); Gy, gray; StDev, standard deviation; h, hour
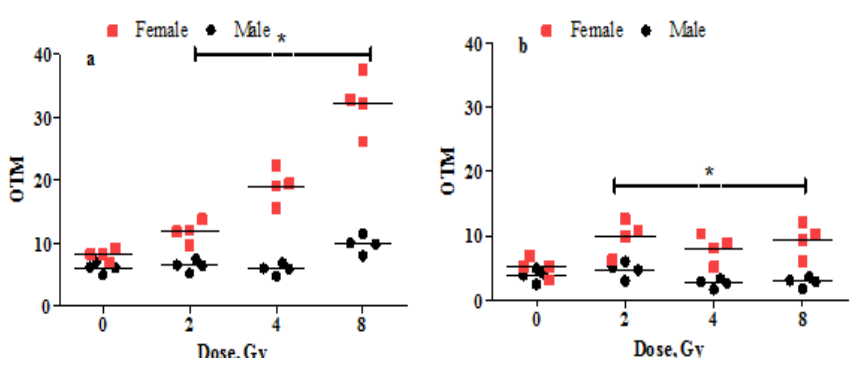

Figure I The dot plot analysis of the relationship between radiosensitivity (DNA damage/repair) and gender of donors. The level of DNA damage was analyzed in peripheral blood mononuclear cells after 0 (a) and 24 (b) hours of irradiation with ultrafast electron beam at the $3,6 \mathrm{~Gy} / \mathrm{min}$ dose-rate; OTM the relative amount of DNA in the tail of the comet multiplied by the median migration distance. ${ }^{*}-\mathrm{p}<0.00 \mathrm{I}$ in comparison between male and female groups.

\section{Acknowledgements}

This work was supported by the RA MES State Committee of Science, in the frames of the research project № 17A-1F008 and ANSEF molbio-4551 grant project.

\section{Conflict of interest}

The authors declare that there are no conflict of interest.

\section{References}

1. Gauduel Y. Laser-Driven Particle Acceleration Towards Radiobiology and Medicine. In: Giulietti A, editor. Laser-Plasma Accelerators Based Ultrafast Radiation Biophysics. Switzerland: Springer; 2016:19-41.
2. Rigaud O, Fortunel NO, Vaigot P, et al. Exploring ultrashort high-energy electron-induced damage in human carcinoma cells. Cell Death Dis. 2010;1:e73.

3. Laschinsky L, Baumann M, Beyreuther E, et al. Radiobiological effectiveness of laser accelerated electrons in comparison to electron beams from a conventional linear accelerator. $J$ Radiat Res. 2012;53(3):395-403.

4. Favaudon V, Caplier L, Monceau V, Pouzoulet F, Sayarath M et al. (2014) Ultrahigh dose-rate FLASH irradiation increases the differential response between normal and tumor tissue in mice. Sci Transl Med. 2014;6(245):254ra93.

5. Babayan N, Hovhannisyan G, Grigoryan B, et al. Dose-rate effect of ultrashort electron beam radiation on DNA damage and repair in vitro. $J$ Radiat Res. 2017;1;58(6):894-897.

6. Alsbeih G, Al-Meer RS, Al-Harbi N, et al. Gender bias in individual radiosensitivity and the association with genetic polymorphic variations. Radiother Oncol. 2016;119(2):236-243.

7. Billings PC, Romero-Weaver AL, Kennedy AR. Effect of Gender on the Radiation Sensitivity of Murine Blood Cells. Gravit Space Res. 2014;2(1):25-31.

8. Preston DL, Shimizu Y, Pierce DA, et al. Studies of mortality of atomic bomb survivors. Report 13: solid cancer and noncancer disease mortality: 1950-1997. Radiat Res. 2003;160(4):381-407.

9. Tsakanov VM, Aroutiounian RM, Amatuni GA, et al. AREAL low energy electron beam applications in life and materials sciences. Nucl Instrum Methods Phys Res. 2016;829:248-253. 